\title{
COMPUTER PREDICTION OF TECHNOLOGICAL REGIMES OF RAPID CONE-SHAPED ADSORPTION FILTERS WITH CHEMICAL REGENERATION OF HOMOGENEOUS POROUS LOADS
}

\author{
Andrii Bomba ${ }^{1}$, Yurii Klymyuk ${ }^{1}$, Ihor Prysiazhniuk ${ }^{2}$ \\ ${ }^{1}$ National University of Water and Environmental Engineering, Department of Applied Mathematics, Rivne, Ukraine \\ ${ }^{2}$ Rivne State University of Humanities, Department of Hanging Mathematics, Rivne, Ukraine
}

Abstract. Mathematical models for predicting technological regimes of filtration (water purification from the present impurities), backwashing, chemical regeneration and direct washing of rapid cone-shaped adsorption filters, taking into account the influence of temperature effects on the internal mass transfer kinetics at constant rates of the appropriate regimes, are formulated. Algorithms for numerical-asymptotic approximations of solutions of the corresponding nonlinear singularly perturbed boundary value problems for a model cone-shaped domain bounded by two equipotential surfaces and a flow surface are obtained. The proposed models in the complex allow computer experiments to be conducted to investigate the change of impurity concentrations in the filtration flow and on the surface of the load adsorbent, temperature of the filtration flow, filtration coefficient and active porosity along the filter height due to adsorption and desorption processes, and on their basis, to predict a good use of adsorbents and increase the protective time of rapid cone-shaped adsorption filters with chemical regeneration of homogeneous porous loads.

Keywords: mathematical model, process of water purification, adsorption, rapid cone-shaped filter, chemical regeneration, homogeneous porous load

\section{KOMPUTEROWE PROGNOZOWANIE TRYBÓW TECHNOLOGICZNYCH SZYBKICH STOŻKOWYCH FILTRÓW ADSORPCYJNYCH Z CHEMICZNĄ REGENERACJĄ JEDNORODNYCH POROWATYCH OBCIĄŻEŃ}

Streszczenie. Sformułowano matematyczne modele do prognozowania trybów technologicznych filtracji (oczyszczanie wody z obecnych zanieczyszczeń), płukania wstecznego, regeneracji chemicznej i bezpośredniego przemywania szybkich stożkowych adsorpcyjnych filtrów z uwzględnieniem wplywu temperatury na kinetykę wewnętrznego przenoszenia masy przy zachowaniu statych prędkości odpowiednich trybów. Opracowano się algorytmy numerycznie asymptotycznych aproksymacji rozwiazań odpowiadajacych problemów nieliniowych pojedynczo zaburzonych brzegowych dla domeny modelu o ksztatcie stożka, ograniczonej dwiema powierzchniami ekwipotencjalnymi i powierzchnia przeptywu. Proponowane modele $w$ kompleksie pozwalaja na prowadzenie eksperymentów komputerowych $w$ celu zbadania zmiany stężeń zanieczyszczeń $w$ strumieniu filtracyjnym $i$ na powierzchni adsorbentu obcią̇ajacego, temperatury przepływu filtracji, współczynnika filtracji oraz porowatości czynnej wzdtuż wysokości filtra ze względu na procesy adsorpcji $i$ desorpcji, na ich podstawie przewidzieć bardziej optymalne zastosowania adsorbentów $i$ wydtużenia czasu ochronnego szybkich stożkowych filtrów adsorpcyjnych z chemiczna regeneracja jednorodnych porowatych obciażen.

Słowa kluczowe: model matematyczny, proces oczyszczania wody, adsorpcja, szybki stożkowy filtr, regeneracja chemiczna, jednorodne porowate obciążenie

\section{Introduction}

Any water needs to be purified before it can be used for domestic and drinking water supply. The main methods of water purification are clarification, decolorization and disinfection. The final stage is its purification from various impurities, in particular, calcium and magnesium salts, the total content of which determines the hardness of the water, as well as iron removal, in rapid adsorption filters with chemical regeneration of porous loads $[4,6]$. They use natural (bentonite, montmorillonite, peat), artificial (activated carbon, artificial zeolites, polysorbs) and synthetic (nanostructured carbon sorbents) materials as adsorbents [17]. The rate of the adsorption process depends on the concentration, nature and structure of the impurities, filtration rate and temperature seepage, and type and properties of the adsorbent [5]. Maintaining a constant set filtration rate is achieved by automatically adjusting the increase in the opening of the valve on the filtrate pipeline as the resistance of the filter load increases due to the accumulation of impurity particles in it. The impulse to increase the opening of the valve on the filtrate pipeline is a change in the water level on the filter (controlled by a float device) or water flow in the filtrate pipeline (controlled by a throttle device and a differential pressure gauge) [11]. When the latch is fully open, the filter is switched off to regenerate the porous load. First, the backwash regime with a high water supply rate (2-3 times higher than the filtration rate), which lasts for 5-20 minutes and allows the filter material of the porous load to loosen and large particles of impurities to be removed. Next, a regime of chemical regeneration is carried out with a high feed rate of a solution of a certain reagent (potassium permanganate $\mathrm{KMnO}_{4}$ is usually used), which starts the process of chemical restoration of the adsorption capacity of the porous load, and lasts for 10-30 minutes. Impurity particles from the filter material pass into the reagent solution. Finally, a regime of direct rinsing at a high water supply rate, lasting up to 10 minutes, seals the filter material of the porous load and removes residues of impurities and the chemical solution of the reagent.

The increasing needs for purified water in industrial enterprises and the growing cost of filter materials require research, on the one hand, into more optimal use of adsorbents and increasing the duration of filters by choosing their shape, in particular, taking into account the influence of changes in the temperature of the filtration flow along the filter height on the process of adsorption water purification, and on the other hand, into restoration of the filtration properties of porous loads by chemical regeneration for their reuse $[4,6]$.

\section{Literature review}

As an analysis of the literature sources shows, in particular $[2,3,5,7,8,12,13,15,16,18,19]$, a significant contribution to the development of the theoretical foundations of filtering liquids through porous loads has been made by many scientists, both domestic and foreign. Note that mathematical models for predicting the technological processes of filtration and regeneration of porous loads by domestic researchers often use the model of D. M. Mintz [15] with constant rates of the respective processes and temperature, or some modification (improved model). In [10], its spatial generalization is proposed to predict the process of water purification from impurities in rapid cone-shaped filters while maintaining a constant filtration rate. The model proposed in this work is more efficient for theoretical studies aimed at optimizing the filtering process parameters (duration, shape, filter size, layer height, etc.) by introducing additional equations to determine the change in active porosity and filtration coefficient of filter load along its height, taking into account diffusion in the filtration flow and on the surface of the load grains. An urgent task is to generalize the appropriate model for computer prediction of technological regimes of filtration, backwashing, chemical regeneration and direct washing of rapid cone-shaped adsorption filters, taking into account the influence 
of temperature effects on the internal mass transfer kinetics at constant rates of the appropriate regimes.

These models in the complex will allow providing computer experiments to predict a better use of adsorbents and increasing the protective time of rapid cone-shaped adsorption filters with chemical regeneration of homogeneous porous loads by taking into account not only the change in the filtration flow rate along the filter height, but also the effect of temperature on the coefficients that characterize the rates of mass transfer during adsorption and desorption, as well as on filtration coefficient.

\section{Formulation of the problem}

Let's develop a model of technological regimes of filtration, backwash, chemical regeneration and direct washing of rapid cone-shaped adsorption filters with chemical regeneration of a homogeneous porous load. We assume that in the filtration regime, the convective components of mass transfer and adsorption outweigh the contribution of diffusion and desorption, and in the backwash, chemical regeneration and direct washing regimes, the convective components of mass transfer and desorption outweigh the contribution of diffusion and adsorption. In addition, due to changes in the temperature of the filtration flow due to adsorption and desorption processes, the influence of temperature effects on the internal kinetics of mass transfer is taken into account. We assume that the convective components of mass transfer and adsorption outweigh the contribution of diffusion and desorption. In addition, the impact of temperature effects on the internal kinetics of mass transfer is taken into account due to changes in the temperature of the filtration flow due to adsorption and desorption processes. So, for the domain $G=G_{\mathrm{z}} \times(0, \infty)$, where $G_{\mathrm{z}}$ is a spatial one-connected domain $(\mathrm{z}=(x, y, z))$ bounded by smooth, orthogonal interconnecting lines, by two equipotential surfaces $S_{*}, S^{*}$ and by the flow surface $S^{* *}$ (Fig. 1), the corresponding spatial model problems for predicting technological regimes of rapid cone-shaped adsorption filters, taking into account the reverse influence of process characteristics (impurity concentration, respectively, in the filtration flow and on the surface of the adsorbent) on the load characteristics (filtration coefficients, porosity, adsorption, desorption) will consist of equations describing the motion of the filtration flow and the equation of continuity:

$$
\left\{\vec{v}=\kappa_{*}^{*} \cdot \operatorname{grad} \varphi, \operatorname{div} \vec{v}=0\right.
$$

Next are equations for determining the change in impurity concentrations in the filtration flow and on the surface of the load adsorbent, temperature of the filtration flow, filtration coefficient and active porosity along the filter height, respectively, for the filtration regime:

$$
\left\{\begin{array}{l}
(\sigma \cdot C)^{\prime}=\operatorname{div}(D \cdot \operatorname{grad} C)-\vec{v} \cdot \operatorname{grad} C- \\
-\alpha \cdot C+\beta \cdot U, \\
(\sigma \cdot U)^{\prime}=\operatorname{div}\left(D^{*} \cdot \operatorname{grad} U\right)+\alpha \cdot C-\beta \cdot U, \\
(\sigma \cdot T)_{t}^{\prime}=\operatorname{div}\left(D^{* *} \cdot \operatorname{grad} T\right)-\vec{v} \cdot \operatorname{grad} T+ \\
+\gamma \cdot(\alpha \cdot C-\beta \cdot U), \\
\kappa_{t}^{\prime}=-\mu \cdot U, \sigma_{t}^{\prime}=-\lambda \cdot U,
\end{array}\right.
$$

backwashing, chemical regeneration and direct washing regimes:

$$
\left\{\begin{array}{l}
(\sigma \cdot C)_{t}^{\prime}=\operatorname{div}(D \cdot \operatorname{grad} C)-\vec{v} \cdot \operatorname{grad} C+ \\
+\beta \cdot U-\alpha \cdot C, \\
(\sigma \cdot U)_{t}^{\prime}=\operatorname{div}\left(D^{*} \cdot \operatorname{grad} U\right)-\beta \cdot U+\alpha \cdot C, \\
(\sigma \cdot T)_{t}^{\prime}=\operatorname{div}\left(D^{* *} \cdot \operatorname{grad} T\right)-\vec{v} \cdot \operatorname{grad} T+ \\
+\gamma \cdot(\beta \cdot U-\alpha \cdot C), \\
\kappa_{t}^{\prime}=\mu \cdot U, \sigma_{t}^{\prime}=\lambda \cdot U,
\end{array}\right.
$$

which are supplemented by the following boundary conditions, respectively, for filtration and direct washing regimes:

$$
\left\{\left.\varphi\right|_{S_{*}}=\varphi_{*},\left.\varphi\right|_{S^{*}}=\varphi^{*},\left.\varphi_{\bar{n}}^{\prime}\right|_{S^{*}}=0\right.
$$

$$
\left\{\begin{array}{l}
\left.C\right|_{S_{*}}=c_{*}^{*},\left.C_{\bar{n}}^{\prime}\right|_{S^{*}}=0,\left.C_{\bar{n}}^{\prime}\right|_{S^{* *}}=0, \\
\left.U\right|_{S_{*}}=u_{*}^{*},\left.U_{\bar{n}}^{\prime}\right|_{S^{*}}=0,\left.U_{\bar{n}}^{\prime}\right|_{S^{* *}}=0, \\
\left.T\right|_{S_{*}}=T_{*}^{*},\left.T_{\bar{n}}^{\prime}\right|_{S^{*}}=0,\left.T_{\bar{n}}^{\prime}\right|_{S^{* *}}=0,
\end{array}\right.
$$

backwash and chemical regeneration regimes:

$$
\begin{aligned}
& \left\{\left.\varphi\right|_{S^{*}}=\varphi_{*},\left.\varphi\right|_{S_{*}}=\varphi^{*},\left.\varphi_{\bar{n}}^{\prime}\right|_{S^{*}}=0,\right. \\
& \left\{\begin{array}{l}
\left.C\right|_{S^{*}}=c_{*}^{*},\left.C_{\bar{n}}^{\prime}\right|_{S_{*}}=0,\left.C_{\bar{n}}^{\prime}\right|_{S^{* *}}=0, \\
\left.U\right|_{S^{*}}=u_{*}^{*},\left.U_{n}^{\prime}\right|_{S_{*}}=0,\left.U_{n}^{\prime}\right|_{S^{* *}}=0, \\
\left.T\right|_{S^{*}}=T_{*}^{*},\left.T_{n}^{\prime}\right|_{S_{*}}=0,\left.T_{n}^{\prime}\right|_{S^{* *}}=0,
\end{array}\right.
\end{aligned}
$$

and initial conditions:

$$
\left\{\begin{array}{l}
\left.C\right|_{t=0}=c_{0}^{0},\left.U\right|_{t=0}=u_{0}^{0},\left.T\right|_{t=0}=T_{0}^{0}, \\
\left.\kappa\right|_{t=0}=\kappa_{0}^{0},\left.\sigma\right|_{t=0}=\sigma_{0}^{0}
\end{array}\right.
$$

where $\varphi=\varphi(x, y, z)$, and $\vec{v}=\vec{v}\left(v_{x}, v_{y}, v_{z}\right)$ is respectively the potential and the velocity vector of the filtration, $0 \leq \varphi_{*}<\varphi<\varphi^{*}<\infty, v=|\vec{v}|=\sqrt{v_{x}^{2}(x, y, z)+v_{y}^{2}(x, y, z)+v_{z}^{2}(x, y, z)}>>0$, $\kappa_{*}^{*}$ is the initial filtration coefficient, $\kappa_{*}^{*}>0, \vec{n}$ is outer normal to the corresponding surface, $C=C(x, y, z, t)$ and $U=U(x, y, z, t)$ are the concentrations of impurities, respectively, in the filtration flow and on the surface of the adsorbent load, $T=T(x, y, z, t)$ is the temperature of the filtration flow at point $(x, y, z)$ at time $t, \kappa=\kappa(x, y, z, t)$ is the filtration coefficient, $\sigma=\sigma(x, y, z, t)$ is the active porosity, $D$ and $D^{*}$ are the impurity diffusion coefficients, respectively, in the filtration flow and on the surface of the adsorbent, $D=\varepsilon \cdot d_{0}, d_{0}>0$, $D^{*}=\varepsilon \cdot d_{0}^{*}, d_{0}^{*}>0, D^{* *}$ is the coefficient of thermal conductivity of the filtration flow, $D^{* *}=\varepsilon \cdot d_{0}^{* *}, d_{0}^{* *}>0, \alpha$ and $\beta$ are coefficients that characterize the rate of mass transfer, respectively, in the adsorption of impurities from the filtration flow on the surface of the load adsorbent and the desorption of impurities from the surface of the load adsorbent into the filtration flow, for the model problem of predicting the filtration regimes $\alpha=\sum_{s_{1}=0}^{2} \sum_{s_{2}=0}^{2-s_{1}} \varepsilon^{s_{1}+s_{2}} \cdot \alpha_{s_{1}, s_{2}} \cdot v^{s_{1}} \cdot T^{s_{2}}, \quad \alpha_{s_{1}, s_{2}} \in \mathrm{R} \quad\left(s_{1}=(0,2)\right.$, $\left.s_{2}=\left(0,2-s_{1}\right)\right), \quad \beta=\varepsilon \cdot \sum_{s_{1}=0}^{2} \sum_{s_{2}=0}^{2-s_{1}} \varepsilon^{s_{1}+s_{2}} \cdot \beta_{s_{1}, s_{2}} \cdot v^{s_{1}} \cdot T^{s_{2}}, \quad \beta_{s_{1}, s_{2}} \in \mathrm{R}$ $\left(s_{1}=(0,2), s_{2}=\left(0,2-s_{1}\right)\right)$ and for the model problems of predicting the backwashing, chemical regeneration and direct washing regimes $\alpha=\varepsilon \cdot \sum_{s_{1}=0}^{2} \sum_{s_{2}=0}^{2-s_{1}} \varepsilon^{s_{1}+s_{2}} \cdot \alpha_{s_{1}, s_{2}} \cdot v^{s_{1}} \cdot T^{s_{2}}, \quad \alpha_{s_{1}, s_{2}} \in \mathrm{R}$ $\left(s_{1}=(0,2), \quad s_{2}=\left(0,2-s_{1}\right)\right), \quad \beta=\sum_{s_{1}=0}^{2} \sum_{s_{2}=0}^{2-s_{1}} \varepsilon^{s_{1}+s_{2}} \cdot \beta_{s_{1}, s_{2}} \cdot v^{s_{1}} \cdot T^{s_{2}}$, $\beta_{s_{1}, s_{2}} \in \mathrm{R} \quad\left(s_{1}=(0,2), s_{2}=\left(0,2-s_{1}\right)\right), \quad \gamma, \mu$ and $\lambda$ are coefficients characterizing the rate of change, respectively, of the filtration flow temperature, filtration coefficient and active porosity due to adsorption and desorption processes, $\mu=\varepsilon \cdot \sum_{s=0}^{2} \varepsilon^{s} \cdot \mu_{s} \cdot T^{s}, \mu_{r, s} \in \mathrm{R}(s=(0,2)), \lambda=\varepsilon \cdot \lambda_{0}$, $\lambda_{0}>0, \quad \alpha=\alpha(x, y, z, t), \quad \beta=\beta(x, y, z, t), \quad \gamma=\gamma(x, y, z, t)$, $\mu=\mu(x, y, z, t)$ are continuous limited functions, $\varepsilon$ is a small parameter $(\varepsilon>0)$ which characterizes the predominance of certain components of the process, $c_{*}^{*}=c_{*}^{*}(x, y, z, t)$, $c_{0}^{0}=c_{0}^{0}(x, y, z), \quad u_{*}^{*}=u_{*}^{*}(x, y, z, t), \quad u_{0}^{0}=u_{0}^{0}(x, y, z)$, $T_{*}^{*}=T_{*}^{*}(x, y, z, t), \quad T_{0}^{0}=T_{0}^{0}(x, y, z), \quad \kappa_{0}^{0}=\kappa_{0}^{0}(x, y, z)$, 
$\sigma_{0}^{0}=\sigma_{0}^{0}(x, y, z)$ are quite smooth functions, consistent with each other on the lines of intersection of surfaces $S_{*}, S^{*}$ and $S^{* *}$ of domain $G$ [1].

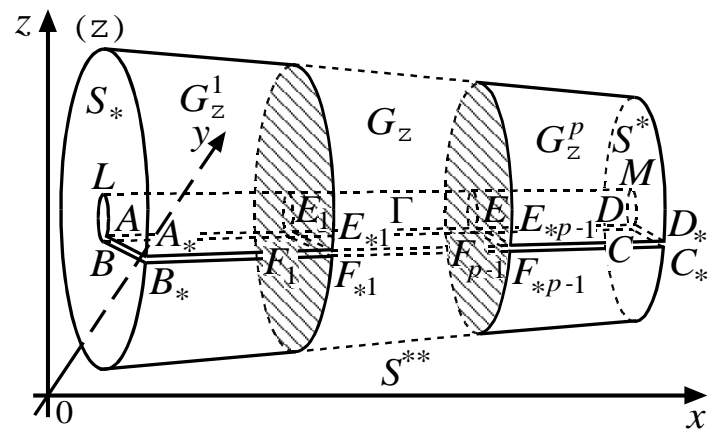

Fig. 1. Spatial filtering domain $G_{\mathrm{z}}$ with conditional section $\Gamma$ (cone-shaped filter)

\section{Materials and methods}

The problem is solved in the same way as in [10] by fixing on the surface $S_{*}$ some point $A(A=B)$ and sequential execution of conditional sections $\Gamma_{1}=A L M D B L M C$ and $\Gamma_{2}=A D D_{*} A_{*} B C C_{*} B_{*}$ along the corresponding surfaces of the flow (we denote for convenience $\Gamma=\Gamma_{1} \cup \Gamma_{2}$ ). The model problems of forecasting of technological regimes of filtration (1), (2), (4), (5), (8), backwash (1), (3), (6)-(8), chemical regeneration (1), (3), (6)-(8) and direct wash (1), (3), (4), (5), (8) in rapid coneshaped filter with chemical regeneration of porous load reduced to the solving of the problems in the received one-connected domain $G_{\mathrm{z}} \backslash \Gamma$ that is a curvilinear parallelepiped $A B C D A_{*} B_{*} C_{*} D_{*}$, bounded by two equipotential surfaces $A B B_{*} A_{*}, C D D_{*} C_{*}$ and four flow surfaces $A B C D=A L M D \cup B L M C, A_{*} B_{*} C_{*} D_{*}$, $A D D_{*} A_{*}=B C C_{*} B_{*}$ (Fig. 1), The surfaces are smooth and orthogonal to each other at angular points and along the edges, with the addition of the impermeability condition $\left.\varphi_{\bar{n}}^{\prime}\right|_{\Gamma}=0$ along section $\Gamma$ :

$$
\begin{aligned}
& \left\{\begin{array}{l}
\left.\varphi\right|_{A B B_{*} A_{*}}=\varphi_{*},\left.\varphi\right|_{C D D_{*} C_{*}}=\varphi^{*}, \\
\left.\varphi_{n}^{\prime}\right|_{A B C D \cup A_{*} B_{*} C_{*} D_{*} \cup A D D_{*} A_{*} \cup B C C_{*} B_{*}}=0,
\end{array}\right. \\
& \left\{\begin{array}{l}
\left.C\right|_{A B B_{*} A_{*}}=C_{*}^{*},\left.C_{\vec{n}}^{\prime}\right|_{C D D_{*} C_{*}}=0, \\
\left.C_{\tilde{n}}^{\prime}\right|_{A D D_{*} A_{*} \cup B C C_{*} B_{*} \cup A B C D \cup A_{*} B_{*} C_{*} D_{*}}=0, \\
\left.U\right|_{A B B_{*} A_{*}}=u_{*}^{*},\left.U_{\vec{n}}^{\prime}\right|_{C D D_{*} C_{*}}=0, \\
\left.U_{\bar{n}}^{\prime}\right|_{A D D_{*} A_{*} \cup B C C_{*} B_{*} \cup A B C D \cup A_{*} B_{*} C_{*} D_{*}}=0, \\
\left.T\right|_{A B B_{*} A_{*}}=T_{*}^{*},\left.T_{\vec{n}}^{\prime}\right|_{C D D_{*} C_{*}}=0, \\
\left.T_{\bar{n}}^{\prime}\right|_{A D D_{*} A_{*} \cup B C C_{*} B_{*} \cup A B C D \cup A_{*} B_{*} C_{*} D_{*}}=0,
\end{array}\right.
\end{aligned}
$$

the initial conditions (8) and the conditions of further "gluing" of the banks of conditional section $\Gamma$ :

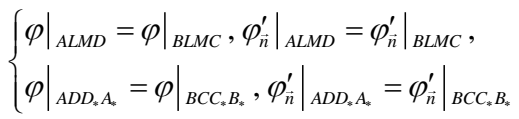

and conditions of agreement of values of impurity concentrations in the filtration flow and on the surface of the load adsorbent and values of the filtration flow temperature on the conditional sections of section $\Gamma$ :

$$
\begin{aligned}
& \left(\left.C\right|_{A L M D}=\left.C\right|_{B L M C},\left.C_{n}^{\prime}\right|_{A L M D}=\left.C_{n}^{\prime}\right|_{B L M C},\right. \\
& \left.C\right|_{A D D_{s} A_{s}}=\left.C\right|_{B C C_{*} B_{s}},\left.C_{\vec{n}}^{\prime}\right|_{A D D_{s} A_{s}}=\left.C_{\vec{n}}^{\prime}\right|_{B C C_{s} B_{s}}, \\
& \left.U\right|_{A L M D}=\left.U\right|_{B L M C},\left.U_{\vec{n}}^{\prime}\right|_{A L M D}=\left.U_{\vec{n}}^{\prime}\right|_{B L M C} \text {, } \\
& \left\{\left.U\right|_{A D D_{*} A_{s}}=\left.U\right|_{B C C_{*} B_{*}},\left.U_{\bar{n}}^{\prime}\right|_{A D D_{s} A_{s}}=\left.U_{\vec{n}}^{\prime}\right|_{B C C_{s} B_{s}},\right. \\
& \left.T\right|_{A L M D}=\left.T\right|_{B L M C},\left.T_{\vec{n}}^{\prime}\right|_{A L M D}=\left.T_{\vec{n}}^{\prime}\right|_{B L M C}, \\
& \left.T\right|_{A D D_{*} A_{*}}=\left.T\right|_{B C C_{*} B_{*}},\left.T_{\bar{n}}^{\prime}\right|_{A D D_{*} A_{*}}=\left.T_{\bar{n}}^{\prime}\right|_{B C C_{*} B_{*}} .
\end{aligned}
$$

Similar to [9], problems (1), (9), (13) and (1), (11), (13) are replaced by the more general direct problem of finding a spatial analogue of the conformal mapping of the one-connected domain $G_{z} \backslash \Gamma$ to the corresponding domain of complex potential which is rectangular parallelepiped $G_{\mathrm{w}}=A^{\prime} B^{\prime} C^{\prime} D^{\prime} A_{*}^{\prime} B_{*}^{\prime} C_{*}^{\prime} D_{*}^{\prime}$ (Fig. 2), where $G_{\mathrm{w}}=\left\{\mathrm{w}=(\varphi, \psi, \eta): \varphi_{*}<\varphi<\varphi^{*}, \quad 0<\psi<Q_{*}\right.$, $\left.0<\eta<Q^{*}\right\}, \quad Q_{*}, \quad Q^{*}$ are unknown parameters, $Q=Q_{*} \cdot Q^{*}$ is the full filtration flow, with subsequent finding of conditions of "gluing" on the banks of conditional section $\Gamma$. The algorithm for solving these problems is obtained in [9], in particular, the velocity field $\vec{v}$, parameters $Q_{*}, Q^{*}, Q$ and a number of other variables are found. By replacing variables $x=x(\varphi, \psi, \eta), y=y(\varphi, \psi, \eta), z=z(\varphi, \psi, \eta)$ in equations (2), (3) and conditions (10), (12), (8), (14), we obtain model problems for predicting the technological regimes of a rapid cone-shaped adsorption filter with chemical regeneration of porous load for the domain $G_{\mathrm{w}} \times(0, \infty)$, described by the systems of equations, respectively, for the filtration regime:

$$
\left\{\begin{array}{l}
(\tilde{\sigma} \cdot c)_{t}^{\prime}=D \cdot\left(b_{1} \cdot c_{\varphi \varphi}^{\prime \prime}+b_{2} \cdot c_{\psi \psi}^{\prime \prime}+b_{3} \cdot c_{\eta \eta}^{\prime \prime}+\right. \\
\left.+b_{4} \cdot c_{\psi}^{\prime}+b_{5} \cdot c_{\eta}^{\prime}\right)-\tilde{v}^{2} / \kappa_{*}^{*} \cdot c_{\varphi}^{\prime}-\tilde{\alpha} \cdot c+\tilde{\beta} \cdot u, \\
(\tilde{\sigma} \cdot u)_{t}^{\prime}=D^{*} \cdot\left(b_{1} \cdot u_{\varphi \varphi}^{\prime \prime}+b_{2} \cdot u_{\psi \psi}^{\prime \prime}+b_{3} \cdot u_{\eta \eta}^{\prime \prime}+\right. \\
\left.+b_{4} \cdot u_{\psi}^{\prime}+b_{5} \cdot u_{\eta}^{\prime}\right)+\tilde{\alpha} \cdot c-\tilde{\beta} \cdot u, \\
(\tilde{\sigma} \cdot \tilde{T})_{t}^{\prime}=D^{* *} \cdot\left(b_{1} \cdot \tilde{T}_{\varphi \varphi}^{\prime \prime}+b_{2} \cdot \tilde{T}_{\psi \psi}^{\prime \prime}+b_{3} \cdot \tilde{T}_{\eta \eta}^{\prime \prime}+\right. \\
\left.+b_{4} \cdot \tilde{T}_{\psi}^{\prime}+b_{5} \cdot \tilde{T}_{\eta}^{\prime}\right)-\tilde{v}^{2} / \kappa_{*}^{*} \cdot \tilde{T}_{\varphi}^{\prime}+\tilde{\gamma} \cdot(\tilde{\alpha} \cdot c-\tilde{\beta} \cdot u), \\
\tilde{\kappa}_{t}^{\prime}=-\tilde{\mu} \cdot u, \tilde{\sigma}_{t}^{\prime}=-\lambda \cdot u,
\end{array}\right.
$$

backwash, chemical regeneration and direct wash regimes:

$$
\left\{\begin{array}{l}
(\tilde{\sigma} \cdot c)_{t}^{\prime}=D \cdot\left(b_{1} \cdot c_{\varphi \varphi}^{\prime \prime}+b_{2} \cdot c_{\psi \psi}^{\prime \prime}+b_{3} \cdot c_{\eta \eta}^{\prime \prime}+\right. \\
\left.+b_{4} \cdot c_{\psi}^{\prime}+b_{5} \cdot c_{\eta}^{\prime}\right)-\tilde{v}^{2} / \kappa_{*}^{*} \cdot c_{\varphi}^{\prime}+\tilde{\beta} \cdot u-\tilde{\alpha} \cdot c, \\
(\tilde{\sigma} \cdot u)_{t}^{\prime}=D^{*} \cdot\left(b_{1} \cdot u_{\varphi \varphi}^{\prime \prime}+b_{2} \cdot u_{\psi \psi}^{\prime \prime}+b_{3} \cdot u_{\eta \eta}^{\prime \prime}+\right. \\
\left.+b_{4} \cdot u_{\psi}^{\prime}+b_{5} \cdot u_{\eta}^{\prime}\right)-\tilde{\beta} \cdot u+\tilde{\alpha} \cdot c, \\
(\tilde{\sigma} \cdot \tilde{T})_{t}^{\prime}=D^{* *} \cdot\left(b_{1} \cdot \tilde{T}_{\varphi \varphi}^{\prime \prime}+b_{2} \cdot \tilde{T}_{\psi \psi}^{\prime \prime}+b_{3} \cdot \tilde{T}_{\eta \eta}^{\prime \prime}+\right. \\
\left.+b_{4} \cdot \tilde{T}_{\psi}^{\prime}+b_{5} \cdot \tilde{T}_{\eta}^{\prime}\right)-\tilde{v}^{2} / \kappa_{*}^{*} \cdot \tilde{T}_{\varphi}^{\prime}+\tilde{\gamma} \cdot(\tilde{\beta} \cdot u-\tilde{\alpha} \cdot c), \\
\tilde{\kappa}_{t}^{\prime}=\tilde{\mu} \cdot u, \tilde{\sigma}_{t}^{\prime}=\lambda \cdot u,
\end{array}\right.
$$


which are supplemented by the following boundary conditions:

$$
\left\{\begin{array}{l}
\left.c\right|_{\varphi=\varphi_{*}}=\tilde{c}_{*}^{*},\left.c_{\varphi}^{\prime}\right|_{\varphi=\varphi^{*}}=0, \\
\left.c_{\psi}\right|_{\psi=0}=\left.c_{\psi}^{\prime}\right|_{\psi=Q_{*}}=\left.c_{\eta}^{\prime}\right|_{\eta=0}=\left.c_{\eta}^{\prime}\right|_{\eta=Q^{*}}=0, \\
\left.u\right|_{\varphi=\varphi_{*}}=\tilde{u}_{*}^{*},\left.u_{\varphi}^{\prime}\right|_{\varphi=\varphi^{*}}=0, \\
\left.u_{\psi}^{\prime}\right|_{\psi=0}=\left.u_{\psi}^{\prime}\right|_{\psi=Q_{*}}=\left.u_{\eta}^{\prime}\right|_{\eta=0}=\left.u_{\eta}^{\prime}\right|_{\eta=Q^{*}}=0, \\
\left.\tilde{T}\right|_{\varphi=\varphi_{*}}=\tilde{T}_{*}^{*},\left.\tilde{T}_{\varphi}^{\prime}\right|_{\varphi=\varphi^{*}}=0, \\
\left.\tilde{T}_{\psi}^{\prime}\right|_{\psi=0}=\left.\tilde{T}_{\psi}^{\prime}\right|_{\psi=Q_{*}}=\left.\tilde{T}_{\eta}^{\prime}\right|_{\eta=0}=\left.\tilde{T}_{\eta}^{\prime}\right|_{\eta=Q^{*}}=0,
\end{array}\right.
$$

initial conditions:

$$
\left\{\begin{array}{l}
\left.c\right|_{t=0}=\tilde{c}_{0}^{0},\left.u\right|_{t=0}=\tilde{u}_{0}^{0},\left.\tilde{T}\right|_{t=0}=\tilde{T}_{0}^{0}, \\
\left.\tilde{\kappa}\right|_{t=0}=\tilde{\kappa}_{0}^{0},\left.\tilde{\sigma}\right|_{t=0}=\tilde{\sigma}_{0}^{0}
\end{array}\right.
$$

and conditions of consistency of the values of impurity concentrations in the filtration flow and on the surface of the load adsorbent and the values of the filtration flow temperature on the conditional surfaces of section $\Gamma$ :

$$
\left\{\begin{array}{l}
\left.c\right|_{\eta=0, \psi=\tilde{\psi}}=\left.c\right|_{\eta=0, \psi=Q_{*}-\tilde{\psi}},\left.c_{\bar{n}}^{\prime}\right|_{\eta=0, \psi=\tilde{\psi}}=\left.c_{\tilde{n}}^{\prime}\right|_{\eta=0, \psi=Q_{*}-\tilde{\psi}}, \\
\left.c\right|_{\psi=0}=\left.c\right|_{\psi=Q_{*}},\left.c_{\tilde{n}}^{\prime}\right|_{\psi=0}=\left.c_{\tilde{n}}^{\prime}\right|_{\psi=Q_{*}}, \\
\left.u\right|_{\eta=0, \psi=\tilde{\psi}}=\left.u\right|_{\eta=0, \psi=Q_{*}-\tilde{\psi}},\left.u_{\tilde{n}}^{\prime}\right|_{\eta=0, \psi=\tilde{\psi}}=\left.u_{\tilde{n}}^{\prime}\right|_{\eta=0, \psi=Q_{*}-\tilde{\psi}}, \\
\left.u\right|_{\psi=0}=\left.u\right|_{\psi=Q_{*}},\left.u_{\tilde{n}}^{\prime}\right|_{\psi=0}=\left.u_{\tilde{n}}^{\prime}\right|_{\psi=Q_{*}}, \\
\left.\tilde{T}\right|_{\eta=0, \psi=\hat{\psi}}=\left.\tilde{T}\right|_{\eta=0, \psi=Q_{*}-\hat{\psi}},\left.\tilde{T}_{\bar{n}}^{\prime}\right|_{\eta=0, \psi=\tilde{\psi}}=\left.\tilde{T}_{\bar{n}}^{\prime}\right|_{\eta=0, \psi=Q_{*}-\hat{\psi}}, \\
\left.\tilde{T}\right|_{\psi=0}=\left.\tilde{T}\right|_{\psi=Q_{*}},\left.\tilde{T}_{\tilde{n}}^{\prime}\right|_{\psi=0}=\left.\tilde{T}_{\tilde{n}}^{\prime}\right|_{\psi=Q_{*}},
\end{array}\right.
$$

where $c=c(\varphi, \psi, \eta, t)=C(x(\varphi, \psi, \eta), y(\varphi, \psi, \eta), z(\varphi, \psi, \eta), t), \ldots$, for the model problem of predicting the filtration regimes: $\tilde{\alpha}=\sum_{s_{1}=0}^{2} \sum_{s_{2}=0}^{2-s_{1}} \varepsilon^{s_{1}+s_{2}} \cdot \tilde{\alpha}_{s_{1}, s_{2}} \cdot \tilde{v}^{s_{1}} \cdot \tilde{T}^{s_{2}}, \quad \tilde{\alpha}_{s_{1}, s_{2}} \in \mathrm{R} \quad\left(s_{1}=(0,2)\right.$, $\left.s_{2}=\left(0,2-s_{1}\right)\right), \quad \tilde{\beta}=\varepsilon \cdot \sum_{s_{1}=0}^{2} \sum_{s_{2}=0}^{2-s_{1}} \varepsilon^{s_{1}+s_{2}} \cdot \tilde{\beta}_{s_{1}, s_{2}} \cdot \tilde{v}^{s_{1}} \cdot \tilde{T}^{s_{2}}, \quad \tilde{\beta}_{s_{1}, s_{2}} \in \mathrm{R}$ $\left(s_{1}=(0,2), s_{2}=\left(0,2-s_{1}\right)\right)$ and for model problems of predicting of backwash, chemical regeneration and direct washing regimes: $\tilde{\alpha}=\varepsilon \cdot \sum_{s_{1}=0}^{2} \sum_{s_{2}=0}^{2-s_{1}} \varepsilon^{s_{1}+s_{2}} \cdot \tilde{\alpha}_{s_{1}, s_{2}} \cdot \tilde{v}^{s_{1}} \cdot \tilde{T}^{s_{2}}, \quad \tilde{\alpha}_{s_{1}, s_{2}} \in \mathrm{R} \quad\left(s_{1}=(0,2)\right.$, $\left.s_{2}=\left(0,2-s_{1}\right)\right), \quad \tilde{\beta}=\sum_{s_{1}=0}^{2} \sum_{s_{2}=0}^{2-s_{1}} \varepsilon^{s_{1}+s_{2}} \cdot \tilde{\beta}_{s_{1}, s_{2}} \cdot \tilde{v}^{s_{1}} \cdot \tilde{T}^{s_{2}}, \quad \tilde{\beta}_{s_{1}, s_{2}} \in \mathrm{R}$ $\left(s_{1}=(0,2), \quad s_{2}=\left(0,2-s_{1}\right)\right), \quad \tilde{\mu}=\varepsilon \cdot \sum_{s=0}^{2} \varepsilon^{s} \cdot \tilde{\mu}_{s} \cdot \tilde{T}^{s}, \quad \tilde{\mu}_{r, s} \in \mathrm{R}$ $(s=(0,2)), \quad b_{1}=\varphi_{x}^{\prime 2}+\varphi_{y}^{\prime 2}+\varphi_{z}^{\prime 2}=\tilde{v}^{2} / \kappa_{*}^{* 2}, \quad b_{2}=\psi_{x}^{\prime 2}+\psi_{y}^{\prime 2}+\psi_{z}^{\prime 2}$, $b_{3}=\eta_{x}^{\prime 2}+\eta_{y}^{\prime 2}+\eta_{z}^{\prime 2}, \quad b_{4}=\psi_{x x}^{\prime \prime}+\psi_{y y}^{\prime \prime}+\psi_{z z}^{\prime \prime}, \quad b_{5}=\eta_{x x}^{\prime \prime}+\eta_{y y}^{\prime \prime}+\eta_{z z}^{\prime \prime}$, $b_{s}=b_{s}(\varphi, \psi, \eta)(s=(1,5)), \tilde{v}=\tilde{v}(\varphi, \psi, \eta), \widehat{\psi} \in\left[0, Q_{*} / 2\right]$.

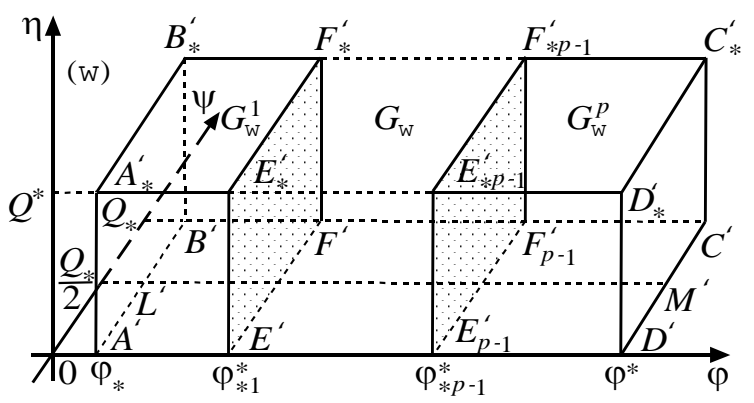

Fig. 2. Spatial domain of complex potential $G_{\mathrm{w}}$

Similar to [10], a numerically asymptotic approximation of the solution $(c, u, \tilde{T}, \tilde{\kappa}, \tilde{\sigma})$ of problems (15), (17)-(19) and (16)-(19) with accuracy $O\left(\varepsilon^{n+1}\right)$ was found in the form of the following series:

$$
\begin{aligned}
& c=\sum_{i=0}^{n} \varepsilon^{i} \cdot c_{i}+\sum_{i=0}^{n+1} \varepsilon^{i} \cdot \sum_{j=1}^{2} P_{1, j, i}+\sum_{i=0}^{n+1} \varepsilon^{i} \cdot \sum_{j=3}^{6} P_{1, j, i}+R_{1, n+1}, \\
& u=\sum_{i=0}^{n} \varepsilon^{i} \cdot u_{i}+\sum_{i=0}^{n+1} \varepsilon^{i} \cdot \sum_{j=1}^{2} P_{2, j, i}+\sum_{i=0}^{n+1} \varepsilon^{i} \cdot \sum_{j=3}^{6} P_{2, j, i}+R_{2, n+1}, \\
& \tilde{T}=\sum_{i=0}^{n} \varepsilon^{i} \cdot \tilde{T}_{i}+\sum_{i=0}^{n+1} \varepsilon^{i} \cdot \sum_{j=1}^{2} P_{3, j, i}+\sum_{i=0}^{n+1} \varepsilon^{i} \cdot \sum_{j=3}^{6} P_{3, j, i}+R_{3, n+1}, \\
& \tilde{\kappa}=\sum_{i=0}^{n} \varepsilon^{i} \cdot \tilde{\kappa}_{i}+\sum_{i=0}^{n+1} \varepsilon^{i} \cdot \sum_{j=1}^{2} P_{4, j, i}+\sum_{i=0}^{n+1} \varepsilon^{i} \cdot \sum_{j=3}^{6} P_{4, j, i}+R_{4, n+1}, \\
& \tilde{\sigma}=\sum_{i=0}^{n} \varepsilon^{i} \cdot \tilde{\sigma}_{i}+\sum_{i=0}^{n+1} \varepsilon^{i} \cdot \sum_{j=1}^{2} P_{5, j, i}+\sum_{i=0}^{n+1} \varepsilon^{i} \cdot \sum_{j=3}^{6} P_{5, j, i}+R_{5, n+1},
\end{aligned}
$$

where $\quad c_{i}=c_{i}(\varphi, \psi, \eta, t), \quad u_{i}=u_{i}(\varphi, \psi, \eta, t), \quad \tilde{T}_{i}=\tilde{T}_{i}(\varphi, \psi, \eta, t)$, $\tilde{\kappa}_{i}=\tilde{\kappa}_{i}(\varphi, \psi, \eta, t), \quad \tilde{\sigma}_{i}=\tilde{\sigma}_{i}(\varphi, \psi, \eta, t) \quad(i=(0, n))$ are members of regular parts of asymptotic, $P_{s, j, i}=P_{s, j, i}\left(\phi_{j}, \psi, \eta, t\right) \quad(s=(0,5)$, $j=(1,2), \quad i=(0, n+1))$ are the boundary layer type functions around $\varphi=\varphi_{*}$ and $\varphi=\varphi^{*} \quad$ (corrections at the entrance to the filter $), P_{s, j, i}=P_{s, j, i}\left(\varphi, \psi_{j-2}, \eta, t\right) \quad(s=(0,5), \quad j=(3,4)$, $i=(0, n+1)), \quad P_{s, j, i}=P_{s, j, i}\left(\varphi, \psi, \eta_{j-4}, t\right) \quad(s=(0,5), \quad j=(5,6)$, $i=(0, n+1))$ are boundary layer type functions, respectively, around $\psi=0, \psi=Q_{*}, \eta=0$ and $\eta=Q^{*}$ (corrections on the side wall of the filter and the shores of conditional section $\Gamma$ ), $\varphi_{1}=\left(\varphi-\varphi_{*}\right) / \varepsilon, \quad \varphi_{2}=\left(\varphi^{*}-\varphi\right) / \varepsilon, \quad \psi_{1}=\psi / \sqrt{\varepsilon}$, $\psi_{2}=\left(Q_{*}-\psi\right) / \sqrt{\varepsilon}, \quad \eta_{1}=\eta / \sqrt{\varepsilon}, \quad \eta_{2}=\left(Q^{*}-\eta\right) / \sqrt{\varepsilon} \quad$ are the corresponding regulatory transformations (stretches), $R_{s, n+1}(\varphi, \psi, \eta, t, \varepsilon) \quad(s=(0,5))$ are the remaining members.

In particular, for $c_{i}, u_{i}, \tilde{T}_{i}, \tilde{\kappa}_{i}, \tilde{\sigma}_{i}(i=0, n)$ of problems (15),

(17)-(19), we obtained the formulas:

$$
\begin{gathered}
c_{0}= \begin{cases}e^{-\tilde{q}_{1}} \cdot\left(\widehat{g}_{0}+\tilde{c}_{*}^{*}(\psi, \eta, t-\tilde{f}(\varphi, \psi, \eta)),\right. & t \geq \tilde{f}, \\
e^{-\tilde{q}_{2}} \cdot\left(\widehat{\widehat{g}}_{0}+\tilde{c}_{0}^{0}\left(\tilde{f}^{-1}(\tilde{f}(\varphi, \psi, \eta)-t, \psi, \eta), \psi, \eta\right)\right), t<\tilde{f},\end{cases} \\
u_{0}=\frac{1}{\tilde{\sigma}_{0}^{0}} \cdot \int_{0}^{t} \tilde{g}_{i}(\varphi, \psi, \eta, \widehat{t}) d \bar{t}+\tilde{u}_{0}^{0},
\end{gathered}
$$$$
\tilde{T}_{0}= \begin{cases}\widehat{\bar{g}}_{0}+\tilde{T}_{*}^{*}(\psi, \eta, t-\tilde{f}(\varphi, \psi, \eta)), & t \geq \tilde{f}, \\ \widehat{\bar{g}}_{0}+\tilde{T}_{0}^{0}\left(\tilde{f}^{-1}(\tilde{f}(\varphi, \psi, \eta)-t, \psi, \eta), \psi, \eta\right), & t<\tilde{f},\end{cases}
$$$$
\tilde{\kappa}_{0}=\tilde{\kappa}_{0}^{0}, \tilde{\sigma}_{0}=\tilde{\sigma}_{0}^{0},
$$$$
c_{i}=\left\{\begin{array}{l}
e^{-\tilde{q}_{1}(\varphi, \psi, \eta, t)} \cdot \widehat{g}_{i}(\varphi, \psi, \eta, t), t \geq \tilde{f}, \\
e^{-\tilde{q}_{2}(\varphi, \psi, \eta, t)} \cdot \widehat{\widehat{g}}_{i}(\varphi, \psi, \eta, t), t<\tilde{f},
\end{array}\right.
$$$$
u_{i}=\frac{1}{\tilde{\sigma}_{0}^{0}} \cdot \int_{0}^{t} \tilde{g}_{i}(\varphi, \psi, \eta, \hat{t}) d \hat{t},
$$$$
\tilde{T}_{i}=\left\{\begin{array}{l}
\hat{\bar{g}}_{i}(\varphi, \psi, \eta, t), t \geq \tilde{f}, \\
\hat{\bar{g}}_{i}(\varphi, \psi, \eta, t), t<\tilde{f},
\end{array}\right.
$$$$
\tilde{\kappa}_{i}=\int_{0}^{t} \breve{g}_{i}(\varphi, \psi, \eta, \hat{t}) d \hat{t},
$$$$
\tilde{\sigma}_{i}=\int_{0}^{t} \breve{g}_{i}(\varphi, \psi, \eta, \widehat{t}) d \widehat{t} \quad(i=\overline{1, n})
$$

where:

$$
\tilde{q}_{1}(\varphi, \psi, \eta, t)=\kappa_{*}^{*} \cdot \tilde{\alpha}_{0,0} \cdot \int_{\varphi_{*}}^{\varphi} \frac{d \widehat{\varphi}}{\tilde{v}^{2}(\hat{\varphi}, \psi, \eta)},
$$

$\tilde{q}_{2}(\varphi, \psi, \eta, t)=\tilde{\alpha}_{0,0} \cdot \int_{0}^{t} \frac{d \hat{\tau}}{\tilde{\sigma}_{0}^{0}\left(\tilde{f}^{-1}(\hat{t}+\tilde{f}(\varphi, \psi, \eta)-t, \psi, \eta), \psi, \eta\right)}$, 


$$
\begin{aligned}
& \widehat{g}_{i}(\varphi, \psi, \eta, t)=\int_{\varphi_{0}}^{\varphi} \frac{g_{i}(\hat{\varphi}, \psi, \eta, \tilde{f}(\hat{\varphi}, \psi, \eta)-\tilde{f}(\varphi, \psi, \eta)+t)}{\tilde{v}^{2}(\hat{\varphi}, \psi, \eta)} \cdot e^{\tilde{\eta}_{1}(\hat{\varphi}, \psi, \eta, t)} d \hat{\varphi}, \\
& \widehat{\hat{g}}_{i}(\varphi, \psi, \eta, t)=\int_{0}^{t} \frac{g_{i}\left(\tilde{f}^{-1}(\hat{t}+\tilde{f}(\varphi, \psi, \eta)-t, \psi, \eta), \psi, \eta, \hat{t}\right)}{\tilde{\sigma}_{0}^{0}\left(\tilde{f}^{-1}(\widehat{t}+\tilde{f}(\varphi, \psi, \eta)-t, \psi, \eta), \psi, \eta\right)} \cdot e^{\tilde{q}_{2}(\varphi, \psi, \eta, \hat{t})} d \hat{t}, \\
& \overline{\bar{g}}_{i}(\varphi, \psi, \eta, t)=\kappa_{*}^{*} \cdot \int_{\varphi_{*}}^{\varphi} \frac{\tilde{\bar{g}}_{i}(\hat{\varphi}, \psi, \eta, \tilde{f}(\hat{\varphi}, \psi, \eta)-\tilde{f}(\varphi, \psi, \eta)+t)}{\tilde{v}^{2}(\hat{\varphi}, \psi, \eta)} d \hat{\varphi}, \\
& \widehat{\overline{\bar{g}}}_{i}(\varphi, \psi, \eta, t)=\int_{0}^{t} \frac{\tilde{\bar{g}}_{i}\left(\tilde{f}^{-1}(\hat{t}+\tilde{f}(\varphi, \psi, \eta)-t, \psi, \eta), \psi, \eta, \hat{t}\right)}{\tilde{\sigma}_{0}^{0}\left(\tilde{f}^{-1}(\hat{t}+\tilde{f}(\varphi, \psi, \eta)-t, \psi, \eta), \psi, \eta\right)} d \widehat{t}, \\
& g_{i}(\varphi, \psi, \eta, t)=I(i, 1) \cdot\left(d _ { 0 } \cdot \left(b_{1} \cdot c_{(i-1) \varphi \varphi}^{\prime \prime}+b_{2} \cdot c_{(i-1) \psi \psi}^{\prime \prime}+\right.\right.
\end{aligned}
$$$$
\left.\left.+b_{3} \cdot c_{(i-1) \eta \eta}^{\prime \prime}+b_{4} \cdot c_{(i-1) \psi}^{\prime}+b_{5} \cdot c_{(i-1) \eta}^{\prime}\right)-\sum_{l=1}^{i}\left(\tilde{\sigma}_{l} \cdot c_{(i-l) t}^{\prime}+\tilde{\sigma}_{l t}^{\prime} \cdot c_{i-l}\right)\right)-
$$$$
-\left(I ( i , 1 ) \cdot \left(\sum_{l=1}^{i} \tilde{\alpha}_{l, 0} \cdot \tilde{v}^{l} \cdot c_{i-l}+\sum_{l=0}^{i-1} \tilde{\alpha}_{0,1} \cdot \tilde{T}_{l} \cdot c_{i-1-l}-\right.\right.
$$$$
\left.-\sum_{l=0}^{i-1} \tilde{\beta}_{l, 0} \cdot \tilde{v}^{l} \cdot u_{i-1-l}\right)+I(i, 2) \cdot\left(\sum_{l=0}^{i-2} \sum_{k=0}^{l} \tilde{\alpha}_{0,2} \cdot \tilde{T}_{k} \cdot \tilde{T}_{l-k} \cdot c_{i-2-l}+\right.
$$$$
\left.+\sum_{l=0}^{i-2} \tilde{\alpha}_{2,2} \tilde{v} \cdot \tilde{T}_{l} \cdot c_{i-2-l}-\sum_{l=0}^{i-2} \tilde{\beta}_{0,1} \cdot \tilde{T}_{l} \cdot u_{i-2-l}\right)+I(i, 3) \times
$$$$
\left.\times\left(\sum_{l=0}^{i-3} \sum_{k=0}^{l} \tilde{\beta}_{0,2} \cdot \tilde{T}_{k} \cdot \tilde{T}_{l-k} \cdot u_{i-3-l}+\sum_{l=0}^{i-3} \tilde{\beta}_{2,2} \cdot \tilde{v} \cdot \tilde{T}_{l} \cdot u_{i-3-l}\right)\right),
$$$$
\tilde{g}_{i}(\varphi, \psi, \eta, t)=\sum_{l=0}^{i} \tilde{\alpha}_{l, 0} \cdot \tilde{v}^{l} \cdot c_{i-l}+I(i, 1) \cdot\left(d _ { 0 } ^ { * } \cdot \left(b_{1} \cdot u_{(i-1) \varphi \varphi}^{\prime \prime}+\right.\right.
$$$$
\left.+b_{2} \cdot u_{(i-1) \psi \psi}^{\prime \prime}+b_{3} \cdot u_{(i-1) \eta \eta}^{\prime \prime}+b_{4} \cdot u_{(i-1) \psi}^{\prime}+b_{5} \cdot u_{(i-1) \eta}^{\prime}\right)-
$$$$
\left.-\sum_{l=1}^{i}\left(\tilde{\sigma}_{l} \cdot u_{(i-l) t}^{\prime}+\tilde{\sigma}_{l t}^{\prime} \cdot u_{i-l}\right)\right)+\left(I ( i , 1 ) \cdot \left(\sum_{l=0}^{i-1} \tilde{\alpha}_{0,1} \cdot \tilde{T}_{l} \cdot c_{i-1-l}-\right.\right.
$$$$
\left.-\sum_{l=0}^{i-1} \tilde{\beta}_{l, 0} \cdot \tilde{v}^{l} \cdot u_{i-1-l}\right)+I(i, 2) \cdot\left(\sum_{l=0}^{i-2} \sum_{k=0}^{l} \tilde{\alpha}_{0,2} \cdot \tilde{T}_{k} \cdot \tilde{T}_{l-k} \cdot c_{i-2-l} \times\right.
$$$$
\left.+\sum_{l=0}^{i-2} \tilde{\alpha}_{2,2} \tilde{v} \cdot \tilde{T}_{l} \cdot c_{i-2-l}-\sum_{l=0}^{i-2} \tilde{\beta}_{0,1} \cdot \tilde{T}_{l} \cdot u_{i-2-l}\right)-I(i, 3) \times
$$$$
\times\left(\sum_{l=0}^{i-3} \sum_{k=0}^{l} \tilde{\beta}_{0,2} \cdot \tilde{T}_{k} \cdot \tilde{T}_{l-k} \cdot u_{i-3-l}+\sum_{l=0}^{i-3} \tilde{\beta}_{2,2} \cdot \tilde{v} \cdot \tilde{T}_{l} \cdot u_{i-3-l}\right),
$$

$\tilde{\bar{g}}_{i}(\varphi, \psi, \eta, t)=\gamma \cdot \sum_{l=0}^{i} \tilde{\alpha}_{l, 0} \cdot \tilde{v}^{l} \cdot c_{i-l}+I(i, 1) \cdot\left(d_{0}^{* *} \cdot\left(b_{1} \cdot \tilde{T}_{(i-1) \varphi \varphi}^{\prime \prime}+\right.\right.$

$\left.+b_{2} \cdot \tilde{T}_{(i-1) \psi \psi}^{\prime \prime}+b_{3} \cdot \tilde{T}_{(i-1) \eta \eta}^{\prime \prime}+b_{4} \cdot \tilde{T}_{(i-1) \psi}^{\prime}+b_{5} \cdot \tilde{T}_{(i-1) \eta}^{\prime}\right)-$

$\left.-\sum_{l=1}^{i}\left(\tilde{\sigma}_{l} \cdot \tilde{T}_{(i-l) t}^{\prime}+\tilde{\sigma}_{l t}^{\prime} \cdot \tilde{T}_{i-l}^{\prime}\right)\right)+\gamma \cdot\left(I(i, 1) \cdot\left(\sum_{l=0}^{i-1} \tilde{\alpha}_{0,1} \cdot \tilde{T}_{l} \cdot c_{i-1-l}-\right.\right.$ $\left.-\sum_{l=0}^{i-1} \tilde{\beta}_{l, 0} \cdot \tilde{v}^{l} \cdot u_{i-1-l}\right)+I(i, 2) \cdot\left(\sum_{l=0}^{i-2} \sum_{k=0}^{l} \tilde{\alpha}_{0,2} \cdot \tilde{T}_{k} \cdot \tilde{T}_{l-k} \cdot c_{i-2-l}+\right.$$$
\left.+\sum_{l=0}^{i-2} \tilde{\alpha}_{2,2} \cdot \tilde{v} \cdot \tilde{T}_{l} \cdot c_{i-2-l}-\sum_{l=0}^{i-2} \tilde{\beta}_{0,1} \cdot \tilde{T}_{l} \cdot u_{i-2-l}\right)-I(i, 3) \times
$$

$$
\left.\times\left(\sum_{l=0}^{i-3} \sum_{k=0}^{l} \tilde{\beta}_{0,2} \cdot \tilde{T}_{k} \cdot \tilde{T}_{l-k} \cdot u_{i-3-l}+\sum_{l=0}^{i-3} \tilde{\beta}_{2,2} \cdot \tilde{v} \cdot \tilde{T}_{l} \cdot u_{i-3-l}\right)\right),
$$

$\breve{g}_{i}(\varphi, \psi, \eta, t)=-\left(I(i, 1) \cdot \tilde{\mu}_{0} \cdot u_{i-1}+I(i, 2) \cdot \sum_{l=0}^{i-2} \tilde{\mu}_{1} \cdot \tilde{T}_{l} \cdot u_{i-2-l}+\right.$

$\left.+I(i, 3) \cdot \sum_{l=0}^{i-3} \sum_{k=0}^{l} \tilde{\mu}_{2} \cdot \tilde{T}_{k} \cdot \tilde{T}_{l-k} \cdot u_{i-3-l}\right), \quad \breve{g}_{i}(\varphi, \psi, \eta, t)=-\lambda_{0} \cdot u_{i-1}$

and to find $c_{i}, u_{i}, \tilde{T}_{i}, \tilde{\kappa}_{i}, \tilde{\sigma}_{i}(i=0, n)$ in problems (16)-(19), the following formulas are obtained:

$$
c_{0}=\left\{\begin{array}{lr}
\widehat{g}_{0}(\varphi, \psi, \eta, t)+\tilde{c}_{*}^{*}(\psi, \eta, t-\tilde{f}(\varphi, \psi, \eta)), & t \geq \tilde{f}, \\
\widehat{\bar{g}}_{0}(\varphi, \psi, \eta, t)+\tilde{c}_{0}^{0}\left(\tilde{f}^{-1}(\tilde{f}(\varphi, \psi, \eta)-t, \psi, \eta), \psi, \eta\right), & t<\tilde{f},
\end{array}\right.
$$

$$
u_{0}=\tilde{u}_{0}^{0} \cdot e^{-\frac{\tilde{\beta}_{0.0} .0}{\tilde{\sigma}_{0}^{0}} \cdot t}
$$

$\tilde{T}_{0}=\left\{\begin{array}{lr}\hat{\bar{g}}_{0}(\varphi, \psi, \eta, t)+\tilde{T}_{*}^{*}(\psi, \eta, t-\tilde{f}(\varphi, \psi, \eta)), & t \geq \tilde{f}, \\ \hat{\bar{g}}_{0}(\varphi, \psi, \eta, t)+\tilde{T}_{0}^{0}\left(\tilde{f}^{-1}(\tilde{f}(\varphi, \psi, \eta)-t, \psi, \eta), \psi, \eta\right), & t<\tilde{f},\end{array}\right.$

$$
\begin{aligned}
& \tilde{\kappa}_{0}=\tilde{\kappa}_{0}^{0}, \tilde{\sigma}_{0}=\tilde{\sigma}_{0}^{0}, \\
& c_{i}=\left\{\begin{array}{l}
\hat{g}_{i}(\varphi, \psi, \eta, t), t \geq \tilde{f}, \\
\hat{\bar{g}}_{i}(\varphi, \psi, \eta, t), t<\tilde{f},
\end{array}\right. \\
& u_{i}=\frac{\tilde{g}_{i}(\varphi, \psi, \eta, t)}{\tilde{\beta}_{0,0}} \cdot\left(1-\cdot e^{-\frac{\tilde{\beta}_{0,0}, t}{\tilde{\sigma}_{0}^{0}}}\right), \\
& \tilde{T}_{i}=\left\{\begin{array}{l}
\hat{\bar{g}}_{i}(\varphi, \psi, \eta, t), t \geq \tilde{f}, \\
\overline{\bar{g}}_{i}(\varphi, \psi, \eta, t), t<\tilde{f},
\end{array}\right. \\
& \tilde{\kappa}_{i}=\int_{0}^{t} \breve{g}_{i}(\varphi, \psi, \eta, \widehat{t}) d \widehat{t}, \\
& \tilde{\sigma}_{i}=\int_{0}^{t} \breve{g}_{i}(\varphi, \psi, \eta, \widehat{t}) d \widehat{t} \quad(i=\overline{1, n}),
\end{aligned}
$$

where:

$\widehat{g}_{i}(\varphi, \psi, \eta, t)=\kappa_{*}^{*} \cdot \int_{\varphi_{*}}^{\varphi} \frac{g_{i}(\hat{\varphi}, \psi, \eta, \tilde{f}(\hat{\varphi}, \psi, \eta)-\tilde{f}(\varphi, \psi, \eta)+t)}{\tilde{v}^{2}(\hat{\varphi}, \psi, \eta)} d \hat{\varphi}$, $\widehat{\widehat{g}}_{i}(\varphi, \psi, \eta, t)=\int_{0}^{t} \frac{g_{i}\left(\tilde{f}^{-1}(\hat{t}+\tilde{f}(\varphi, \psi, \eta)-t, \psi, \eta), \psi, \eta, \hat{t}\right)}{\tilde{\sigma}_{0}^{0}\left(\tilde{f}^{-1}(\widehat{t}+\tilde{f}(\varphi, \psi, \eta)-t, \psi, \eta), \psi, \eta\right)} d \hat{t}$, $\overline{\bar{g}}_{i}(\varphi, \psi, \eta, t)=\kappa_{*}^{*} \cdot \int_{\varphi_{*}}^{\varphi} \frac{\tilde{g}_{i}(\hat{\varphi}, \psi, \eta, \tilde{f}(\hat{\varphi}, \psi, \eta)-\tilde{f}(\varphi, \psi, \eta)+t)}{\tilde{v}^{2}(\widehat{\varphi}, \psi, \eta)} d \widehat{\varphi}$, $\hat{\overline{\bar{g}}}_{i}(\varphi, \psi, \eta, t)=\int_{0}^{t} \frac{\tilde{\bar{g}}_{i}\left(\tilde{f}^{-1}(\hat{t}+\tilde{f}(\varphi, \psi, \eta)-t, \psi, \eta), \psi, \eta, \hat{t}\right)}{\tilde{\sigma}_{0}^{0}\left(\tilde{f}^{-1}(\hat{t}+\tilde{f}(\varphi, \psi, \eta)-t, \psi, \eta), \psi, \eta\right)} d \bar{t}$, $g_{i}(\varphi, \psi, \eta, t)=\sum_{l=0}^{i} \tilde{\beta}_{l, 0} \cdot \tilde{v}^{l} \cdot u_{i-l}+I(i, 1) \cdot\left(d_{0} \cdot\left(b_{1} \cdot c_{(i-1) \varphi \varphi}^{\prime \prime}+\right.\right.$ $\left.+b_{2} \cdot c_{(i-1) \eta \mu}^{\prime \prime}+b_{3} \cdot c_{(i-1) \eta \eta}^{\prime \prime}+b_{4} \cdot c_{(i-1) \psi}^{\prime}+b_{5} \cdot c_{(i-1) \eta}^{\prime}\right)-$ $\left.-\sum_{l=1}^{i}\left(\tilde{\sigma}_{l} \cdot c_{(i-l) t}^{\prime}+\tilde{\sigma}_{l t}^{\prime} \cdot c_{i-l}\right)\right)+\left(I(i, 1) \cdot\left(\sum_{l=0}^{i-1} \tilde{\beta}_{0,1} \cdot \tilde{T}_{l} \cdot u_{i-1-l}-\right.\right.$ $\left.-\sum_{l=0}^{i-1} \tilde{\alpha}_{l, 0} \cdot \tilde{v}^{l} \cdot c_{i-1-l}\right)+I(i, 2) \cdot\left(\sum_{l=0}^{i-2} \sum_{k=0}^{l} \tilde{\beta}_{0,2} \cdot \tilde{T}_{k} \cdot \tilde{T}_{l-k} \cdot u_{i-2-l}+\right.$ $\left.+\sum_{l=0}^{i-2} \tilde{\beta}_{2,2} \cdot \tilde{v} \cdot \tilde{l}_{l} \cdot u_{i-2-l}-\sum_{l=0}^{i-2} \tilde{\alpha}_{0,1} \cdot \tilde{T}_{l} \cdot c_{i-2-l}\right)-I(i, 3) \times$ $\times\left(\sum_{l=0}^{i-3} \sum_{k=0}^{l} \tilde{\alpha}_{0,2} \cdot \tilde{T}_{k} \cdot \tilde{T}_{l-k} \cdot c_{i-3-l}+\sum_{l=0}^{i-3} \tilde{\alpha}_{2,2} \cdot \tilde{v} \cdot \tilde{T}_{l} \cdot c_{i-3-l}\right)$, $\tilde{g}_{i}(\varphi, \psi, \eta, t)=I(i, 1) \cdot\left(d_{0}^{*} \cdot\left(b_{1} \cdot u_{(i-1) \varphi \varphi}^{\prime \prime}+b_{2} \cdot u_{(i-1) \psi \psi}^{\prime \prime}+\right.\right.$ $\left.\left.+b_{3} \cdot u_{(i-1) \eta \eta}^{\prime \prime}+b_{4} \cdot u_{(i-1) \psi}^{\prime}+b_{5} \cdot u_{(i-1) \eta}^{\prime}\right)-\sum_{l=1}^{i}\left(\tilde{\sigma}_{l} \cdot u_{(i-l) t}^{\prime}+\tilde{\sigma}_{l t}^{\prime} \cdot u_{i-l}\right)\right)-$ $-\left(I(i, 1) \cdot\left(\sum_{l=1}^{i} \tilde{\beta}_{l, 0} \cdot \tilde{v}^{l} \cdot u_{i-l}+\sum_{l=0}^{i-1} \tilde{\beta}_{0,1} \cdot \tilde{T}_{l} \cdot u_{i-1-l}-\right.\right.$ $\left.-\sum_{l=0}^{i-1} \tilde{\alpha}_{l, 0} \cdot \tilde{v}^{l} \cdot c_{i-1-l}\right)+I(i, 2) \cdot\left(\sum_{l=0}^{i-2} \sum_{k=0}^{l} \tilde{\beta}_{0,2} \cdot \tilde{T}_{k} \cdot \tilde{T}_{l-k} \cdot u_{i-2-l}+\right.$ $\left.+\sum_{l=0}^{i-2} \tilde{\beta}_{2,2} \cdot \tilde{v} \cdot \tilde{T}_{l} \cdot u_{i-2-l}-\sum_{l=0}^{i-2} \tilde{\alpha}_{0,1} \cdot \tilde{T}_{l} \cdot c_{i-2-l}\right)+I(i, 3) \times$ $\left.\times\left(\sum_{l=0}^{i-3} \sum_{k=0}^{l} \tilde{\alpha}_{0,2} \cdot \tilde{T}_{k} \cdot \tilde{T}_{l-k} \cdot c_{i-3-l}+\sum_{l=0}^{i-3} \tilde{\alpha}_{2,2} \cdot \tilde{v} \cdot \tilde{T}_{l} \cdot c_{i-3-l}\right)\right)$, $\tilde{\bar{g}}_{i}(\varphi, \psi, \eta, t)=\tilde{\gamma} \cdot \sum_{l=0}^{i} \tilde{\beta}_{l, 0} \cdot \tilde{v}^{l} \cdot u_{i-l}+I(i, 1) \cdot\left(d_{0}^{* *} \cdot\left(b_{1} \cdot \tilde{T}_{(i-1) \varphi \varphi}^{\prime \prime}+\right.\right.$ $\left.+b_{2} \cdot \tilde{T}_{(i-1) \psi \psi}^{\prime \prime}+b_{3} \cdot \tilde{T}_{(i-1) \eta \eta}^{\prime \prime}+b_{4} \cdot \tilde{T}_{(i-1) \psi}^{\prime}+b_{5} \cdot \tilde{T}_{(i-1) \eta}^{\prime}\right)-$ $\left.-\sum_{l=1}^{i}\left(\tilde{\sigma}_{l} \cdot \tilde{T}_{(i-l) t}^{\prime}+\tilde{\sigma}_{l t}^{\prime} \cdot \tilde{T}_{i-l}^{\prime}\right)\right)+\gamma \cdot\left(I(i, 1) \cdot\left(\sum_{l=0}^{i-1} \tilde{\beta}_{0,1} \cdot \tilde{T}_{l} \cdot u_{i-1-l}-\right.\right.$ $\left.-\sum_{l=0}^{i-1} \tilde{\alpha}_{l, 0} \cdot \tilde{v}^{l} \cdot c_{i-1-l}\right)+I(i, 2) \cdot\left(\sum_{l=0}^{i-2} \sum_{k=0}^{l} \tilde{\beta}_{0,2} \cdot \tilde{T}_{k} \cdot \tilde{T}_{l-k} \cdot u_{i-2-l}+\right.$ $\left.+\sum_{l=0}^{i-2} \tilde{\beta}_{2,2} \cdot \tilde{v} \cdot \tilde{T}_{l} \cdot u_{i-2-l}-\sum_{l=0}^{i-2} \tilde{\alpha}_{0,1} \cdot \tilde{T}_{l} \cdot c_{i-2-l}\right)-I(i, 3) \times$ 


$$
\begin{gathered}
\left.\times\left(\sum_{l=0}^{i-3} \sum_{k=0}^{l} \tilde{\alpha}_{0,2} \cdot \tilde{T}_{k} \cdot \tilde{T}_{l-k} \cdot c_{i-3-l}+\sum_{l=0}^{i-3} \tilde{\alpha}_{2,2} \cdot \tilde{v} \cdot \tilde{T}_{l} \cdot c_{i-3-l}\right)\right) \\
\breve{g}_{i}(\varphi, \psi, \eta, t)=I(i, 1) \cdot \tilde{\mu}_{0} \cdot u_{i-1}+I(i, 2) \cdot \sum_{l=0}^{i-2} \tilde{\mu}_{1} \cdot \tilde{T}_{l} \cdot u_{i-2-l}+ \\
+I(i, 3) \cdot \sum_{l=0}^{i-3} \sum_{k=0}^{l} \tilde{\mu}_{2} \cdot \tilde{T}_{k} \cdot \tilde{T}_{l-k} \cdot u_{i-3-l}, \bar{g}_{i}(\varphi, \psi, \eta, t)=\lambda_{0} \cdot u_{i-1}, \\
\tilde{f}=\tilde{f}(\varphi, \psi, \eta)=\kappa_{*}^{*} \cdot \int_{\varphi_{*}}^{\varphi} \frac{\tilde{\sigma}_{0}^{0}(\hat{\varphi}, \psi, \eta)}{\tilde{v}^{2}(\hat{\varphi}, \psi, \eta)} d \hat{\varphi} \text { is the time of passing }
\end{gathered}
$$

of the respective particles of the impurity from point $\quad\left(x\left(\varphi_{*}, \psi, \eta\right), y\left(\varphi_{*}, \psi, \eta\right), z\left(\varphi_{*}, \psi, \eta\right)\right) \in G_{z} \quad$ to point $(x(\varphi, \psi, \eta), y(\varphi, \psi, \eta), z(\varphi, \psi, \eta)) \in G_{z}, \tilde{f}^{-1}$ is function inverted according to $\tilde{f}$ with respect to variable $\varphi, I(a, b)= \begin{cases}1, & a \geq b, \\ 0, & a<b .\end{cases}$

\section{Conclusions}

The mathematical models for predicting the technological regimes of filtration (water purification from the present impurities), backwashing, chemical regeneration and direct washing of rapid cone-shaped adsorption filters, taking into account the influence of temperature effects on the internal mass transfer kinetics at a constant rate of the appropriate regimes, have been formed. Algorithms for numerical-asymptotic approximations of solutions of the corresponding nonlinear singularly perturbed boundary value problems for a model coneshaped domain bounded by two equipotential surfaces and a flow surface have been obtained under the condition that in the filtration regime, the convective components of mass transfer and adsorption outweigh the contribution of diffusion and desorption, and in the backwashing of chemical regeneration and direct washing regimes, the convective components of mass transfer and desorption outweigh the contribution of diffusion and adsorption. The proposed models in the complex allow computer experiments to be conducted to investigate the change of impurity concentrations in the filtration flow and on the surface of the load adsorbent, the temperature of the filtration flow, the filtration coefficient, and the active porosity along the filter height due to adsorption and desorption processes, and on their basis good use of adsorbents to be predicted, and the protective time of rapid cone-shaped adsorption filters with chemical regeneration of homogeneous porous loads be increased.

\section{References}

[1] Bomba A. Ya., Klymiuk Yu. Ye.: Mathematical modeling of spatial singularperturbed filtration-convection-diffusion processes. TzOV firma "Assol", Rivne 2014.

[2] Bomba A. Ya., Prysiazhniuk I. M., Prysiazhniuk O. V.: Methods of perturbation theory for predicting heat transfer processes in porous and microporous media. O. Zen, Rivne 2017

[3] Bomba A. Ya., Safonyk A. P.: Modeling of nonlinearly perturbed processes of purification of liquids from multicomponent contaminants. NUVHP, Rivne 2017.

[4] Edzwald J.: Water Quality \& Treatment. A Handbook on Drinking Water, McGraw-Hill Professional, 2010.

[5] Heikkinen M., Poutiainen H., Liukkonen M., Heikkinen T., Hiltunen Y.: Selforganizing maps in the analysis of an industrial wastewater treatment process. Mathematics and Computers in Simulation 82(3), 2011, 450-459.

[6] Hendricks D. W.: Fundamentals of water treatment unit processes: physical, chemical, and biological. CRC Press, Boca Raton 2011.

[7] Ives K. J.: Deep-bed water filters. New developments. Filtr. and Separ. 6(1), 1969, 42-48.

[8] Kalteh A. M., Hjorth P., Berndtsson R.: Review of the self-organizing map (SOM) approach in water resources: analysis, modelling and application. Environmental Modelling and Software 23(7), 2008, 835-845.

[9] Klimjuk Ju. Je.: Construction of filtration fields for rapid filters conical shape with homogeneous porous loads. Proceedings of II International scientific conference "World Science in 2016: Results". Lulu Press, Morrisville 2017, 95 99.

[10] Klimjuk Ju. Je.: Prediction of changes in the characteristics of filter materials in rapid cone-shaped waterpurifying filters. Innovative solutions in modern science $8(27), 2018,72-84$.

[11] Nevzorova A.B.: Fundamentals of automation of water supply and sanitation. UO BelGUT, Gomel 2005.

[12] Maleki A., Pajootan E., Hayati B.: Ethyl acrylate grafted chitosan for heavy metal removal from wastewater: Equilibrium, kinetic and thermodynamic studies. Journal of the Taiwan Institute of Chemical Engineers 51(0), 2015, 127 134.

[13] Maier H. R., Dandy G. C.: Neural networks for the prediction and forecasting of water resources variables: a review of modelling issues and applications. Environmental Modelling and Software 15(1), 2000, 101-124.

[14] Makarevich N. A., Bogdanovich N. I.: The theoretical basis of adsorption. SAFU, Arkhangelsk 2015.

[15] Mincz D. M.: Theoretical foundations of water purification technology. Strojizdat, Moscow 1964

[16] Safonyk A. P.: Modelling the filtration processes of liquids from multicomponent contamination in the conditions of authentication of mass transfer coefficient. International Journal of Mathematical Models and Methods in Applied Sciences 9, 2015, 189-192.

[17] Sakalova H. V., Vasylinych T. M.: Investigation of the efficiency of purification of heavy metal ions from wastewater using natural adsorbents. TOV Tvory, Vinnytsia 2019.

[18] Vries D., Bertelkamp C., Schoonenberg Kegel F., Hofs B., Dusseldorp J., Bruins J. H., de Vet W., van den Akker B.: Iron and manganese removal: Recent advances in modelling treatment efficiency by rapid sand filtration. Water Research 109, 2017, 35-45.

[19] Zevi Y., Dewita S., Aghasa A., Dwinandha D.: Removal of Iron and Manganese from Natural Groundwater by Continuous Reactor Using Activated and Natural Mordenite Mineral Adsorption. International IOP Conf. Ser., Earth Environ. Sci, $111,2018,012016,1-7$

\section{Prof. Andrii Ya. Bomba}

e-mail: abomba@ukr.net

Professor of the Department Computer Science and Applied Mathematics of the National University of Water and Environmental Engineering, Rivne, Ukraine.

$\mathrm{He}$ is the author of over 450 scientific works, including 9 monographs. A well-known specialist in mathematical modeling and computational methods. Academician of the UNGA. Member of four editions of collections of scientific works and two Specialized Scientific Councils for the defense of theses.

http://orcid.org/0000-0001-5528-4192

\section{Ph.D. Yurii Ye. Klymyuk}

e-mail:klimyuk@ukr.net

Associate professor of the Department Computer Science and Applied Mathematics of the National University of Water and Environmental Engineering, Rivne, Ukraine.

Engaged in scientific mathematical modeling of natural and technological processes, computer simulation of technological processes, computer techniques and computer technologies, programming. http://orcid.org/0000-0003-3672-8469

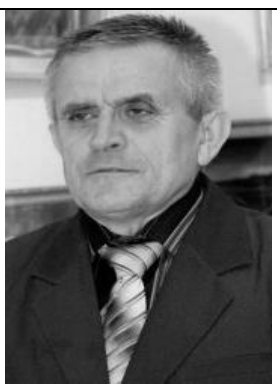

\section{Ph.D. Ihor M. Prysiazhniuk}

e-mail: igorpri79@gmail.com

Associate professor of the Department of Mathematics of Rivne State University of Humanities, Rivne, Ukraine.

Engaged in applied and computational mathematics, mathematical modeling of technological processes, numerical modeling and analysis, differential equations in applied mathematics, physics and engineering, computer simulation.

http://orcid.org/0000-0003-4531-1788

otrzymano/received: 3.08 .2020 przyjęto do druku/accepted: 10.12 .2020 